\title{
Hacking the Body
}

\author{
Camille Baker \\ Digital Media, Engineering and Design \\ Brunel University, Uxbridge \\ United Kingdom \\ camille@swampgirl67.net
}

\author{
Kate Sicchio \\ Lincoln School of Performing Arts \\ University of Lincoln, Lincoln \\ United Kingdom \\ kate@sicchio.com
}

\begin{abstract}
This paper looks at the technological practice of hacking and how choreography and participatory performance can be shaped by its ethos, methods, and approaches. The authors discuss how their current work explores emerging technological tools and devices to find new ways to devise performances to make immersive experiences for audiences. We use hacking culture and theory as a theoretical framework to connect technology and performance ideologies and critically examine key thinkers and practitioners from both domains. This not only informs our own practice and research, but also provides an alternate process for others attempting to bridge these fields.
\end{abstract}

Hacking. Biosensing. Performance. Mobile visualisations.

\section{INTRODUCTION}

Hacking the Body is a new collaborative research project that explores using the concept of 'hacking' to re-purpose and re-imagine internal signals from the body. The project is two-fold: (1) it explores how internal physiological data can be gathered and harnessed to understand the experiential states of the body, and then (2) how we as artists will 'hack' to explore new methods for creating artworks, using sensing systems and audiovisual technology. This will result in works such as sitespecific performances, mobile installations, and participatory performance experiences.

A much-misused term (Jordan, 2008), hacking is typically incorrectly connected to controversial technical practices of 'cracking' into systems and causing damage or stealing financial or sensitive information. However, traditionally the hacker has referred to a technical elite whose prowess has been displayed through the imaginative repurposing of consumer electrical equipment and computing that is the more accurate definition of hacking. Once feared for its lack of adherence to social norms (Soderberg, 2008), the hacker open, hands-on ethos is increasingly finding favour in all manner of creative collaborations.

We term our proposed artistic outcomes as hacks partly because we will be deliberately repurposing existing technologies as part of the technological innovation (biofeedback sensors with mobile computing devices), but also because we understand the human body as an essential component in the global communication network, with its own internal network to be hacked. Our objective is to explore ways in which human physical states can be meaningfully exposed in the network and repurposed, thereby 'hacking the body'. By exploring the use of mobile technologies and the states of the body, new communications technologies practices and artistic work are created.

Mark Deuze states throughout his book Media Life (2012) that we are media and our bodies are already mediatised. He goes onto quote Eugene Thacker who says "...that there exists some fundamental equivalency between genetic 'codes' and computer 'codes'... "(Thacker, 2004:5 in Deuze, 2012:95). This concept of the body as code reflects the possibilities that hacking may afford in this project - what else can this code run? Within the artworks we are currently developing, biological code will be hacked to co-create externally projected and mobile visualisations via custom applications that help expose the inner workings of the body in less literal and medical means, and more interactive aesthetic means.

Hacking the Body and its exploration of the body and technology draws on an existing performance and electronic arts research (Khut, 2011; Benford and Giannachi, 2011; Chatzichristodoulou et al, 2009; Schiphorst, 2007), but extends this in various directions, particularly in the use of open source mobile applications in combination with physiological sensors in participatory artworks, (Donnarumma, 2012; Farnham, 2012; Baker, 2010; Kozel, 2008) to reconceptualise the practice of 
hacking. This combination of different disciplines will help to further understand the relationship between communications technologies and the body. Hacking the Body will identify ways to bridge these gaps and delve between and around these layers of new technological advancements through the use of a hacking methodology.

Since the introduction of the iPhone, the speed of advances of application-based technologies has exponentially developed, opening up much more powerful and significantly improved possibilities for innovation and artistic work. Sensor devices, smart textiles, as well as electronic technologies have also become increasingly cable-free, WiFi-enabled, smaller, and more powerful, through rapid development in materials. In addition, recent commercial movement sensing interfaces for gaming, such as the Kinect, Wii and OmniTouch have resulted in an explosion of hacks and innovative uses in entertainment and artworks. We are using these technologies in various combinations, as well as making our own interactive, mobile visualisation applications using open-source platforms such as Processing, Arduino, and/or openFrameworks. These artworks will be developed as a result of hacking.

\section{HACKING AS METHODOLOGY}

Hacker culture creates the methodology that is employed in Hacking the Body. This draws on various discourses around hacking to help further hacking as a way in which it can be utilised as a collaborative understanding of working with art and technology.

Hacking culture itself has many subcultures, strands, and definitions ranging from open source and free software communities to those creating viruses or attempting to circumvent security. Within the context of this project, a hack is defined to be "a material practice that produces differences in computer, network and communications technologies" (Jordan, 2008:12). While hacking may be seen as re-purposing or subverting data, code or other information, it can also be seen as a re-understanding of what is possible. There are also political and social agendas that are associated with hacking. Specifically in Hacking the Body, hacker ethics are concerned primarily with sharing, openness, collaboration, and engaging in the hands-on imperative.

We also aim to problematise the concept of computer hacking and exploiting it for reuse in examining the flesh of body systems, to create a new hybrid definition. Hacking the Body will thus be developing its own methods to repurpose a hacking' ethos for use within performance and art practices, using body sensing technology to expose inner code of the human body, as discussed by Thacker and Deuze (2012). A performance hacking methodology shifts concepts such as code, networks and technology to extend into new parameters, such as inner and outer states of the human body. Body information can then be hacked and re-purposed, and re-visualised within performative works.

Three hacks or performative experiments take different approaches to using technology within performances to engage audience/ participants in the biological and emotional states of their bodies. By repurposing emerging communications technologies, and 'hacking' body code to devise choreographed and participatory performances, new insights into body response and behaviour, and how human biological code can be represented and then reused will emerge. These hacks are outlined below. By allowing the public to engage with these body-data based performances and installations, they will engage with their physical existence differently.

Beyond the practicalities of redirecting the functionality of technology, there is also the ethos of hacking which is often collaborative but also transdisciplinary in its approaches. "Hackers have constituted an expansive pragmatic practice of instrumental yet playful experimentation and production. In these activities the lines between play, exploration, pedagogy and work are rarely rigidly drawn" (Coleman, 2013, p. 99). This is key to the methodology in Hacking the Body, where participatory performance and exploration 'user workshops' are key to working with technology and the body.

We envision new insights into the understanding of the body and how we represent and re-purpose our own biological code. "As part of this practical capacity, the very nature of hacking - turning a system against itself - is the processing of using existing code, comments, and technology for more than what the original authors intended" (Coleman, 2013 , p. 98). By allowing the public to engage with the performances and installations, they will be able to explore their own physical existence within new artistic and interactive contexts.

\section{THREE HACKS THE CHARM}

Hacking the Body uses the open, solution-driven, hands-on ethos as its main definition and driving principle of authoring creative works. By the means of biofeedback sensing and actuating devices, smartphones, motion sensing or tracking interfaces, and augmented reality tools, this project will develop applications and interactive systems 
for artworks. The project involves three hacks, which each describe and apply aspects of capturing, exploring, and interpreting the inward unseen states of the body, further feeding them outward into public artworks.

(1) Hack one "inside out" - biosensing: breath, blood volume, chemical. This exploration examines the inner flow of fluids in the body, and the conceptual interpretations and representations (journeys, roads, networks, conduits, rivers). This hack explores the methods of reading the internal values of the body and sending the physiological data outward to a mobile device for visualisation. Physiological sensors, which measure inner bodily regulatory systems, such as blood volume and pressure (BVP) or hemoencephalography (HEG) measuring brain blood flow. Other possibilities include breath, heart rate (EEG, EMG or EKG), brainwaves, or skin conductance, using biochemical sensing devices such as non-invasive (saliva sensors) and other blood glucose meters. Custom visualisations or mobile applications detect the states of a performer's body, to show body data for participant interaction and display to the audience through personal displays (their own smartphones) to provide a uniquely engaging experience. A focus on developing garments embedded with the most appropriate wearable physiological sensing devices and textiles has been established through the development of this first hack.

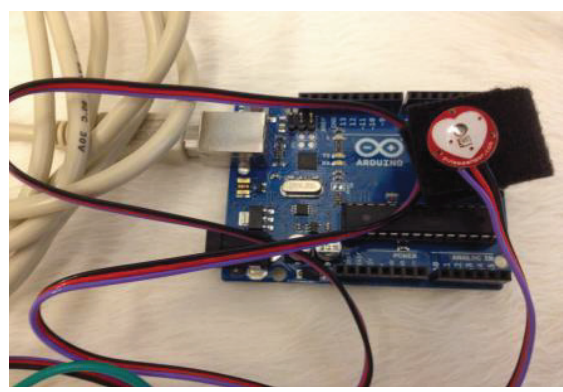

Figure 1: Arduino with Pulse Sensor - one of the approaches to biosensing with DIY electronics by Kate Sicchio 2013

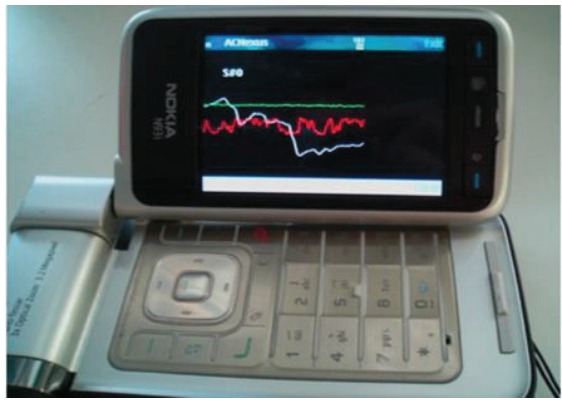

Figure 2: Explorations with body data capture on mobile phone for MINDtouch project by Camille Baker 2008
(2) Hack two, "outside in" - movement visualisation to actuation on the body. This hack uses video tracking to acquire information of movement, gestures and external aspects of the body. The outward states of the body reflect the interior states of the body as an organism, may often be described as sensations. To expose these immeasurable inner qualities, this workshop repurposes the measured external aspects of the body. It applies video sensing systems, such as the Xbox Kinect, mobile implementations of visual tracking, or accelerometers to drive the content of a public, interactive video system.

The notion of the boundaries or the threshold of the skin guides this hack. Here we link gesture to emotions of the participant, studying and exploring more authentic expression versus staged movement, especially gesture and non-verbal communication. This behaviour is then translated, responded to, and hacked. The resulting movement is translated into visualisations and haptic actuation with wearable devices for movers to respond to and create new movement. The actuations, code, instructions and responses become the choreography. Movement will be developed in conjunction with somatic practices and interactive technologies using a collaborative hacking methodology. This part of the project uses a range of hacked devices to capture gestures, movement, and non-verbal interaction. Actuating devices (servos and motors) and emerging technologies are used with textiles and newly developed biosimilar interfaces (such as fabrics made from milk solids, or fungus, etc.).

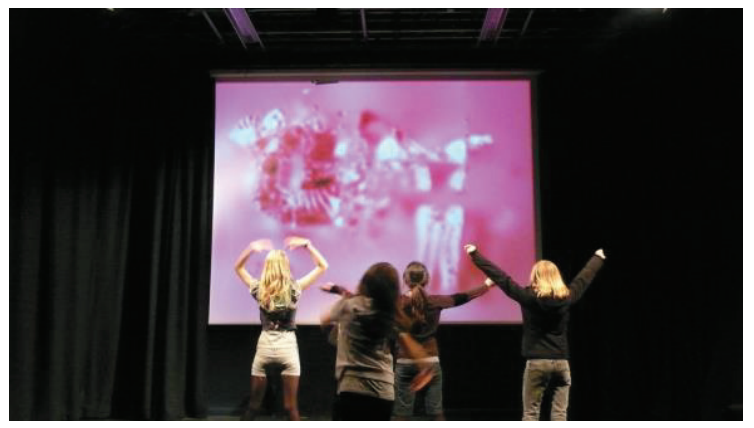

Figure 3: Previous video sensing installation by Kate Sicchio 2007

(3) Hack three "inside matching outside" mobile Artificial Reality applications, QR codes, and projection mapping. This hack explores the relationships between inwards-directed hack one and outwards-oriented hack two. It focuses on the conceptual notion of 'the reveal', such as revealing the shadow layers of the self, drawn from Indian yogic philosophy and psychology. It also draws on the phenomenological aspects of revealing body codes. This hack focuses on understanding, how the inner and outer states of the body connect, and 
how they can be harnessed to create participatory experiences. Participants will interact with others through a mixed reality (multi-user phone-based augmented reality, QR codes and projection mapping), aiming to create a feedback loop between the body's internal data and outside movement, interaction and body expressivity, back to the body and others' bodies. The hack engages participants to use their own body through breath or gestural actions, as well as their emotional responses to these physiological states. One approach involves scanning the body with a custom mobile AR app to reveal body code or project it onto a nearby wall. The mobile device could act as a light that reveals body code, dissolving the shadow of the participant. It will also explore how participants can connect to others, so participants across the globe can connect, share and actively control the collective experience, creating a unique dynamic between the audience and the performers/participants, freed from the bounds of geography.

All three hacks use the methodology of hacking in a collaborative environment to develop a generalisable 'hack-based' working practice. This proposal seeks to explore ways in which the development of such technical exploits can be democratised though a close collaboration of technical and artistic practitioners, specifically through this series of 'body hacks'.
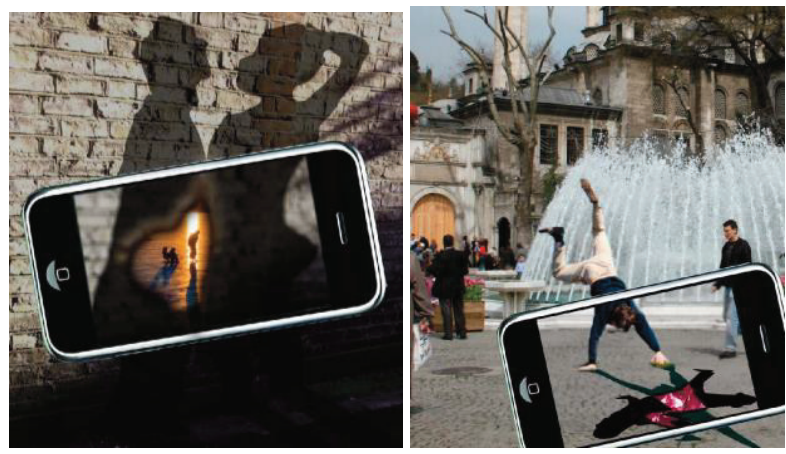

Figure 4 and 5: Artistic visions of playful gestural explorations of body 'data' and augmented reality $(A R)$ image by Kasia Molga with Camille Baker 2012

The project's challenges lie in its unique way of coupling art and technology under the concept of 'hacking the body'. All three 'hacks' will contribute to both disciplines: working together in creating participatory artworks allows firstly, discovering new ways of using the mobile and sensing technologies, and, secondly, by improving user engagement and adding new meaning to digital performances and the body by using personal devices beyond the original purposes. The project will provide new insights into designing robust, accessible, and aesthetic interfaces, as well as the plethora artistic and general public, to express the physiological states of the body in simple, playful, and captivating ways.

\section{BODY HACKING AND FUTURE MOBILE VISUALISATIONS}

Portable and increasingly ubiquitous forms of mobile media and communication devices have transformed the ways in which we transmit our affective and emotional "states," predominantly via text and language. However, they do not yet effectively capture the many layers of non-verbal expression that contribute to communication. One aspect of Hacking the Body is exploring ways to bridge these gaps and delve between and around these layers. The use of mobile technology in participatory performance and participatory cinema activities provides a fresh approach to a still very niche area of art practice and research.

While the reconceptualisation of mobile devices here is novel, the advances in technology within this field are central to the unique aspects of the project. Since the introduction of the iPhone, the speed of advances of application-based and networking technologies has exponentially developed, opening up much more powerful and significantly improved possibilities for innovation and artistic work. Smart phones are now developed to enable complex projects, new options involving real-time interactivity and complex visualisation, due to their speed and the ubiquity of open-source, cross-platform inter-operability, and ease of realtime data transfer, across global networks, using social media tools to reach a variety of audiences.

The smartphone has given rise to the appearance of a new type of audience, readily equipped with powerful tools to experience and get involved in innovative interactive formats, possibly on a global scale. Sensor devices and electronic technologies have also become increasingly cable-free, WiFienabled, smaller, more powerful, and there has been rapid and extensive development in electronic materials, conductive inks and threads, as well as smart textiles in general for use in various fashion, medical and military, as well as artistic projects.

The development of video sensing, on-body and other gestural interfaces by Nintendo and Microsoft and others, such as the Wii, Kinect and recent OmniTouch have also resulted in an explosion of innovative uses, including entertainment, education and art projects. Visual detection algorithms on mobile phones are becoming more powerful, leading to a smarter interpretation of live camera images, and thus the space around us. Visualisation, spatial annotation or 3D placement of additional layers of content has seen major 
development too, with Google Glass. These technologies are ripe for exploitation for use in Hacking the Body.

The relevance of the project is that it has the foresight to wed emerging fields of communication technologies, participatory installation, cinema and performance, to those used by commercial realm and mainstream society. The development of these tools, foresees follow-on research that may lead to bodily functions someday being available and readable on one's phones, just like the weather forecast, which could have further impact for medical or social research. By shifting the concept of the codes and networks of the body into the communication technologies context, where information can be hacked and re-purposed, we are revisualising how people relate to their own body data as code to be hacked.

Three hacks within this project allow for different approaches to technology educating people on the insides of our bodies and how they relate to the world around us. One of the concrete objectives, in addition to aiding in further understanding of functions in the body, is to help people understand communication technologies better in its daily role in our lives. By taking communication technologies out of their current uses, and creating participatory performances with the body, new insights for the use of communication technologies will emerge.

\section{CONCLUSION}

The metaphor of Hacking the Body provides a new vision for hacking in reference to participatory performance and process driven works, while expanding the use of emerging biosensing, wearable and mobile technology. This exploration in hacking body data for artworks, aims to enable greater public engagement in body-based artworks. Hacking the Body' explores the concept, ethos and practices from computer hacking in the context of manipulation and examination of the body through a sequence of three 'hacks' of body code to create performances, installations and mobile applications, using collaborative and open-source approaches. Exposing the human form and physiological states in more scintillating and engaging ways will connect the inner body network to the wider global communications network, by hacking and repurposing 'code'.

\section{REFERENCES}

Auslander, P. (1999) Liveness: Performance in a Mediatized Culture, Routledge, London and New York.

Baker, C.C. (2010) MINDtouch-Ephemeral Transference: Liveness' in Networked Performance with Mobile Devices, PhD Thesis, University of East London Library, London, UK, December 2010.

Coleman, E.G. (2013) Coding Freedom: The Ethics and Aesthetics of Hacking. Princeton University Press, Princeton and Oxford.

Dueze, M. (2012) Media Life, Cambridge, UK, Polity Press.

Donnarumma, M. (2012) Biotechnological Performance Practice / Pratiques de performance biotechnologique. in eContact! Vol 14.2.

Idhe, D. (2002) Bodies in Technology, Electronic Mediations Vol. 5, University of Minnesota Press, Minneapolis, MN.

Jordan, T. (2008) Hacking: Digital Media and Technological Determinism, Digital Media and Society, Polity, Cambridge, UK.

Khut, G. (2006) Development and Evaluation of Participant-centred Biofeedback Artworks, Doctoral thesis. University of Western Sydney, Australia.

Kozel, S. (2007) Closer: Performance, Technologies, Phenomenology, The MIT Press, Cambridge, MA.

Mitchell, W. (2003) Me++: The Cyborg Self and the Networked City, MIT Press, Cambridge, MA.

Massumi, B. (2002) Parables for the Virtual Movement, Affect, Sensation. Durham and London: Duke University Press.

Soderberg, J. (2008) Hacking Capitalism. The Free and Open Source Software Movement. Routledge.

Schiphorst, T. (2007) The Varieties of User Experience: Bridging Embodied Methodologies from Somatics and Performance to Human Computer Interaction, PhD Dissertation, Plymouth, UK: CAiiA Star at the University of Plymouth. 